\title{
ANALYSIS OF SOM \& SOFM TECHNIQUES USED IN SATELLITE IMAGERY
}

\author{
1Rachita Sharma, 2Sanjay Kumar Dubey \\ 1GGIT, Gr. Noida, India \\ E-Mail: rachita.sharma.cs@gmail.com \\ 2Amity University, Noida, India \\ E-Mail: skdubey1@amity.edu
}

\begin{abstract}
This paper describes the introduction of Supervised and Unsupervised Techniques with the comparison of SOFM (Self Organized Feature Map) used for Satellite Imagery. In this we have explained the way of spatial and temporal changes detection used in forecasting in satellite imagery. Forecasting is based on time series of images using Artificial Neural Network. Recently neural networks have gained a lot of interest in time series prediction due to their ability to learn effectively nonlinear dependencies from large volume of possibly noisy data with a learning algorithm. Unsupervised neural networks reveal useful information from the temporal sequence and they reported power in cluster analysis and dimensionality reduction. In unsupervised learning, no pre classification and pre labeling of the input data is needed. SOFM is one of the unsupervised neural network used for time series prediction .In time series prediction the goal is to construct a model that can predict the future of the measured process under interest. There are various approaches to time series prediction that have been used over the years. It is a research area having application in diverse fields like weather forecasting, speech recognition, remote sensing. Advances in remote sensing technology and availability of high resolution images in recent years have motivated many researchers to study patterns in the images for the purpose of trend analysis.
\end{abstract}

Keywords: SOFM, Satellite Imagery, Artificial Neural Network, Supervised Learning and Unsupervised learning.

\section{INTRODUCTION}

Based on the way of learning, all Artificial Neural Networks can be classified in two learning techniques i.e. Supervised Learning and Unsupervised Learning [1]. In supervised learning, a desired output result for each input vector is required when the network is trained and in unsupervised learning, the training of the network is entirely data-driven and no target results for the input data vectors are provided. In terms of Artificial Neural Network, supervised learning uses the target result to guide the formation of the neural parameters such as multi layer perception and unsupervised learning can be used for clustering the fetched data and calculate features inherent to the problem such as the self organizing map.

Based on unsupervised learning which stands for no human intervention is required during the learning. Only small knowledge needs about the characteristics of the input data [2]. Self organize map uses the clustering of data without knowing the class memberships of the input data. Self organize map can also be used for feature detection inherent to the problem, so it is also called self organized feature map (SOFM). It provides a topology preserving mapping from high dimension space to map units (neurons). Topology Preserving described as mapping preserves the relative distance between the points. Points nearer to each other in the input space are mapped to nearby map units in the self organize map (SOM). The SOM serve as the cluster analyzing tool of high dimension data and SOM has the capability to generalize the data.

\section{SOM ALGORITHM}

It basically has two phases: Training and Testing. In Training, first it selects output layer topology, secondly train weights connecting inputs to outputs. Topology is used in conjunction with current mapping of inputs to outputs, to define which weights will be updated. Distance Measure using the topology is reduced over the time and reduces the number of weights that get updated per iteration [4]. So leaning rate is also updated over time. In Testing, It uses weights from training. Network Architecture is shown in figure 1 and complete algorithm of SOM is explained below:

Algo: Self Organize Map

$$
\begin{aligned}
& \text { Select output layer network topology } \\
& \text { Initialize current neighborhood distance, } \mathrm{D}(0) \text {, to a positive value } \\
& \text { Initialize weights from inputs to outputs to small random values Let } \mathrm{t}=1 \\
& \text { While computational bounds are not exceeded do }
\end{aligned}
$$

Step 1: Select an input sample iो

Step 2: Compute the square of the Euclidean distance of $i_{1}$ from weight vectors (wj) associated with each output node

$$
\sum_{k=1}^{n}\left(i_{i \alpha}-w_{j, k}(t)\right)
$$


Step 3: Select output node $j^{*}$ that has weight vector with minimum value from step 2.

Step 4: Update weights to all nodes within a topological distance given by $\mathrm{D}(\mathrm{t})$ from $j^{\star}$, using the weight update rule

$$
w_{j}(t+1)=w_{f}(t)+\eta(t)\left(t_{i}-w_{j}(t)\right)
$$

Step 5: Increment $\mathrm{t}$

end while

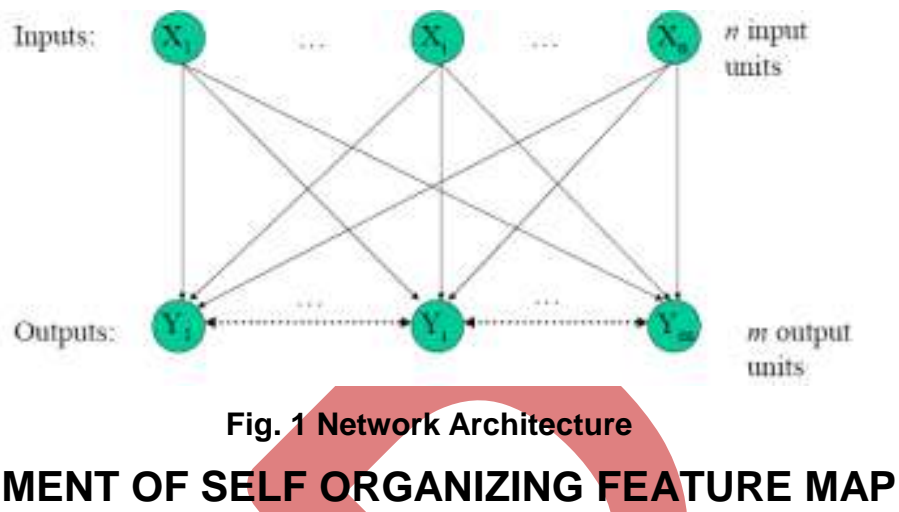

\section{ACCURACY ASSESSMENT OF SELF ORGANIZING FEATURE MAP}

A self organizing feature map is one of the artificial neural networks based on unsupervised learning. It is characterized by the formation of topographic map of the input patterns in which the spatial locations (i.e. coordinates) of the neurons in the lattice are indicative of intrinsic statistical features contained in the input patterns, hence the name -self organizing map[5]. It is the vector quantization method with topology preservation [6]. The training algorithm of the SOFM is based on unsupervised learning, where one sample, the input pattern $x(n)$ from the input space $V_{1}$ is selected randomly and compared against the weight vector $w_{i}$ of the unit $i$ in the map space $V_{M}$ The best matching unit $b$ to given input pattern $x(n)$ is selected using some metric based criterion, such as-

$$
\left\|x(n)-w_{b}\right\|=\min _{i} \in V_{M}\left\{\left\|x-w_{i}(n)\right\|\right\}
$$

Where || || denote the Euclidean vector norm. Initially all weight vectors are set randomly to their initial positions in the input space. During the learning phase the weights in the map are updated towards the given input pattern $x(n)$ according to

$$
w_{i}(n+1)=w_{i}(n)+\gamma(n) h_{i b}(n)\left(x(n)-w_{i}(n)\right)
$$

where $i \in V_{M}$ and $\gamma(n), 0<=\gamma(n)<=1$, is a scalar valued adaptation gain. The neighborhood function $h_{i b}(n)$, gives the excitation of unit $i$ when the best matching unit is $b$. If the map is trained properly, i.e. the gain and the neighborhood function are properly decreased over training a mapping is formed, where weight vector specify centers of clusters satisfying the vector quantization criteria

$$
\left.E=\min \left\{\sum_{j=1}^{M}\left|x_{j}-\mathrm{w}_{\mathrm{b}(\mathrm{x}) \mathrm{j}}\right|\right]\right\}
$$

Where we seek to minimize the sum squared distance $E$ of all input patterns $x_{j}, j=1,2 \ldots . M$, to the respective best matching units with weight vectors $\mathrm{w}_{\mathrm{b}}(\mathrm{xj})[6,7]$.

\section{DETECT CHANGES IN SATELLITE IMAGERY}

In SOFM several improvements is made to learn to recognize temporal changes in a data taken at different time. One of them is Temporal Kohonen Map (TKM) [5]. TKM differs from the SOFM only in its output. The outputs of the normal SOFM are reset to zero after presenting each input pattern and selecting the best matching unit. Hence the map is sensitive to last input pattern. TKM will fire all of its neurons to varying degree of excitation. The output of each neuron is a leaky integrator unit, so there is a time delay involved.

Change detection is a process of showing changes between two images of the same area, collected at different times. Typically, pixel-by-pixel comparisons are made and output is generated when corresponding pixels have sufficiently different grey value. To detect the change in the given image of different time we first extract feature of given image. Feature extraction is a process of extracting features from imagery, such as roads, railways, and water bodies that can be displayed on maps or in Geographic Information System (GIS). On the basis of that feature we compare two images and detect the change [8].

In the earth sciences, digital satellite images have become an important source of information. These images are taken at various temporal resolutions. A satellite image is not a photograph taken by a camera with film in it but is an image. All Commercial satellites acquired with digital sensors. The sensor has thousands of tiny detectors that measure the amount of electromagnetic radiation reflecting from earth surface and object on it. These are called Spectral Measurements. Each spectral reflectance value is recorded as a digital number. These numbers are transmitted back to Earth where they are converted by computer into colors or gray scale converts they brightness levels to create an image that look like a 
photograph. Depending on the desired sensitivity of the detectors, sensor can measure reflectance of energy in the visible, near infrared, thermal infrared, and microwave radar portions of the EM spectrum [9]. Most remote sensing satellite measures energy in very specific well defined wavelength of the spectrum.

Multispectral satellite images are acquired by a digital sensor that measures reflectance in many bands. For instance, one set of detector may measure reflected visible red energy, while another set may measure near infrared energy. These multiple reflectance values are combined to create color images. Current multispectral remote sensing satellites measure reflectance in three to seven different bands at once measure near infrared energy. These multiple reflectance values are combined to create color images. Current multispectral remote sensing satellites measure reflectance in three to seven different bands at once [10].

\section{CONCLUSION}

So the classifications for satellite imagery using self organizing feature map have been done. The classification difference taken over a period of time can be used for trend analysis using SOFM. [11]. Such techniques can indeed be applied for a variety of purposes such as deforestation, archeology, urban planning and development, damage assessment, defense intelligence, and environmental monitoring, weather forecasting etc.

\section{REFERENCES}

[1] G. A. Carpenter, "The ART of adaptive pattern recognizing neural Network", IEEE Comput. Mag., pp. 77-88, Mar. 1988.

[2] A. B. Yegnarayana, "Artificial Neural Networks", Prentice Hall of India Pvt. Ltd, New Delhi, 1999.

[3] R. C. Gonzalez, "Richard E Woods Digital Image Processing", Pearson Education, Inc Second Edition.

[4] H. S. Chae, S. J. Kim and J. A, Rye, "A Classification of MultiSpectral Landsat TM Data using Principal Component Analysis and ANN”, IEEE 1997.

[5] N. R. Euliano and J. C. Principe. "Self- Spatio Temporal Self Organising Feature Map", Computational NeuroEngineering Laboratory, Department of Electrical Engineering, University of Florida, Gainesville, FL 32611.

[6] A. Mangal, P. Mathur and R. Govil. "Trend Analysis in satellite Imagery Using SOFM". Apaji Institute of Mathematics \& Computer Technology, Banasthali Vidhyapith, Rajasthan, India.

[7] A.S.Weigend and N.Gershenfeld, editors. Time series prediction: "forecasting the future and understanding the past", Wesley, 1993.

[8] T.Koskela, M. Varsta, J.Heikkonen, and K. Kaski. "Time series prediction using recurrent SOM with local linear models. Int. J. of knowledge-Based Intelligent Engineering System",Volume 2, Issue 1, pp 60-68,1998.

[9] H. Simon, "Neural Network: A comprehensive Foundation”, Prentice Hall, 1994

[10] G. J. Chappell , J. G. Taylor, "The temporal Kohonen map, Neural Networks", Volume 6 Issue 3, pp 441-445, 1993.

[11] P. Sharma and U. Mutreja, "Analysis of Satellite Images using Artificial Neural Network", International Journal of Soft Computing and Engineering (IJSCE) ISSN: 2231-2307, Volume-2, Issue-6, January 2013.

\section{Author's biography}

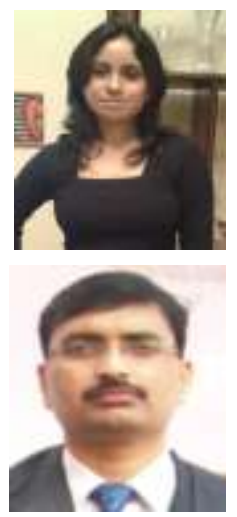

Rachita Sharma is lecturer in GNIT Girls Institute of Technology, Gr. Noida and pursuing M.Tech. from Amity University, Noida in the field of Time Variant Change Analysis in Satellite Imagery. She has also published three papers in International journals and international conferences.

Mr. Sanjay Kumar Dubey is Assistant Professor and Proctor in Amity University, Uttar Pradesh, India He has submitted his Ph. D. thesis in Object Oriented Software Engineering. He has published more than 73 papers in International Journals. He has presented 14 research papers at various National/International conferences. He is member of IET and IEANG. His research areas include Human Computer Interaction, Soft Computing, and Usability Engineering. 